CERN-TH/97-11

KEK-CP052, KEK preprint-166

\title{
Four-quark final state in $W$-pair production: Case of signal and background ${ }^{\dagger}$
}

\author{
T. Ishikawa, Y. Kurihara \\ National Laboratory for High Energy Physics, Oho 1-1, Tsukuba, Ibaraki 305, \\ Japan \\ M. Skrzypek ${ }^{\star}, \mathrm{Z}$. Wa̧s ${ }^{\star} \bullet$ \\ CERN, Theory Division, Geneva 23, Switzerland, \\ and \\ Institute of Nuclear Physics, Kraków, ul. Kawiory 26a, Poland
}

\begin{abstract}
We discuss theoretical predictions for $W$-pair production and decay at LEP2 and higher energies in a form suitable for comparison with raw data. We present a practical framework for calculating uncertainties of predictions given by the KORALW and grc4f Monte Carlo programs. As an example we use observables in the $s \bar{s} c \bar{c}$ decay channel: the total four-quark (four-jet) cross section and two-quark/jet invariant-mass distribution and cross section, in the case when the other two may escape detection.

Effects of QED bremsstrahlung, effective couplings, running $W$ and $Z$ widths, Coulomb interaction and the complete tree level set of diagrams are discussed.

We also revisit the question of technical precision of the new version 1.21 of the KORALW Monte Carlo code as well as of version 1.2(26) of the grc4f one.

Finally we find predictions of the two programs to have an overall physical uncertainty of $2 \%$.

As a side result we show, on the example of an $s \bar{s}$ invariant mass distribution, the strong interplay of spin correlations and detector cut-offs in the case of four-fermion final states.
\end{abstract}

$\dagger$ Work supported in part by Polish Government grants KBN 2P30225206, 2P03B17210, IN2P3 French-Polish Collaboration through LAPP Annecy and European Commission contract ERBCIPDCT940016.

* www home page at http://hpjmiady.ifj.edu.pl/

- Supported at the time of performing part of this study by a Monbusho stipend at the National Laboratory for High Energy Physics, Oho 1-1, Tsukuba, Ibaraki 305, Japan.

CERN-TH/97-11

KEK-CP052, KEK preprint-166

January 1997 


\section{Introduction}

In the summer of 1996 LEP started to collect data in the new centre-of-mass energy zone corresponding to the $W$-pair production threshold and above. During the preparation to the first phase of LEP operation [1], at centre-of-mass energies comparable to the $Z$ mass, it was advocated that different classes of radiative corrections may turn out to be essential in the interpretation of experimental results. Even though it is generally accepted that, if possible, a purely analytical approach is more convenient in comparisons of theoretical predictions with data, in many cases such as a $\tau$ polarization measurement [2], Monte Carlo (MC) modelling of the observables, including the complicated interrelation of experimental cuts and strongly peaked multiphoton phasespace can be handled only with the help of a high-precision MC simulation. In the case of the luminosity measurement at LEP1 [3], QED corrections constitute, even today, the systematic uncertainty surpassing the experimental error and limiting the physical significance of the measurement of, for instance, the number of neutrino species.

It is thus of high practical importance to ensure that a similar situation will not repeat itself at LEP2 and, if necessary, to take appropriate steps in advance. For this purpose a whole family of MC programs and semi-analytical calculations are being developed. A comprehensive report on the status of this work can be found in the report of the LEP2 Workshop [4].

Ultimately in $W$-pair production and decay we expect to reach an experimental precision of order 1\%. ${ }^{1}$ To ensure that uncertainties of theoretical predictions will be negligible, it is thus necessary that they do not surpass $0.5 \%$ and preferably are limited by a $0.3 \%$ threshold.

The purpose of the present paper is therefore to propose a working scheme of estimating these uncertainties. Specifically, we want to supplement technical precision tests of the MC programs with a discussion of the physical precision. For the KORALW version 1.02 [5] it is performed [6] at the technical precision level of better than $0.1 \%$ (in fact better than $0.01 \%$ ) for the $W$-resonant diagrams. Similar tests for the MC program grc4f [7] were performed at the $0.2 \%$ level [4] on the total cross sections for the $W$-resonant diagrams. This technical precision can be improved just by spending a lot of computing time for the simulation as it comes from the statistics of generating events for the comparison. This way, having specified both technical and physical precision of the MC calculation, one will be able to establish the total theoretical error of the results.

We will discuss the general strategy of estimating the physical precision as well as illustrate it on the following observables, all in the $s \bar{s} c \bar{c}$ decay channel: the total fourquark (four-jet) cross section, two-quark/jet invariant-mass distribution and cross section in the case when the other two may escape detection. We will discuss in turn effects such as Coulomb correction, running $W$ and $Z$ widths, effective coupling

\footnotetext{
${ }^{1}$ One has to remember here that 'universal' experimental precision does not exist. It depends on the measured quantities.
} 
constants, etc. We will also comment on the uncertainty related to the remaining classes of electroweak (including QED) corrections, but we will leave aside questions related to QCD final-state corrections and hadronization phenomena.

Since the publication of Refs. [6, 4] which were based on the version 1.03 of the KORALW code, the KORALW program has undergone a series of upgrades, resulting in the recent version $1.21^{2}$. The largest improvements are two sets of complete tree level amplitudes for all the possible massive CC-type final states generated by GRACE packages versions 1 and 2 [8], interfaced to the KORALW generator as optional external matrix elements (amplitudes of GRACE v. 2 are similar to those implemented in the grc4f), along with a new internal presampler to facilitate the general fourfermion phase-space generation. For cross-checks the CC-03 differential distributions and total cross sections of Ref. [6] were reproduced with the help of this new presampler of KORALW. This technical comparison with semi-analytical integration gave an agreement at the level of $0.03 \%$ at least.

The grc4f has a kinematical mapping routine, which can treat the singular behaviour of four-fermion amplitudes, and the numerical integration is done by BASES [9]; on the other hand, the unweighted event generation is performed by SPRING [9]. Therefore by comparing the results from these two packages, the technical test of the independent part of the two packages, i.e. the numerical integration part, was performed. The gauge-parameter independence of the GRACE amplitudes has been confirmed at randomly selected phase points up to the quadruple precision of the variables. Further comparison was carried out with the results of the algebraic manipulation package CompHEP $[10,11]$ for the various four-fermion final states and a precision of at least 8 digits was found for series of randomly chosen points in the phase space.

To cross-check the KORALW and grc4f programs, we compare in Table 1 the numerical results for total cross-section for various input parameter configurations, as described in the table caption and later in the text. This constitutes a significant test of the two programs, as agreement is whenever expected better than $0.3 \%$. The remaining numerical results of the paper are produced by KORALW and cross-checked by grc4f wherever possible.

Finally, there is one more highlight in this paper. In the course of various fourfermion simulations we found an unexpected and strong artificial effect due to the interplay of spin correlations and, seemingly simple, cut-offs on four-fermion phase space. We will illustrate these effects with the example of fake peaks in two-quark invariant-mass distributions.

The layout of the paper is as follows. In the present Sect. 1 we have already discussed the technical tests of the codes. In Sect. 2 we define our observables and elaborate on the question of physical precision. In Sect. 3 we discuss and illustrate the spin correlations issue. We conclude our paper in Sect. 4.

\footnotetext{
${ }^{2}$ Available from http://hpjmiady.ifj.edu.pl/programs/programs.html
} 


\section{Numerical results and general scheme of calculating uncertainty}

To begin with, we want to stress that in principle nothing like an overall uncertainty of the MC program can be defined. For any new observable, one has to repeat anew the numerical analysis as presented here. Nonetheless, from the point of view of presenting a general scheme of how to calculate the uncertainty of the program, the choice of observable is secondary.

For the purpose of the present discussion we choose the $c \bar{c} s \bar{s}$ final state (CC-43 type process) of $W$-pair decay because of its relatively high cross section. We take the following observables: (i) total "visible" $\left(\sigma_{4}\right)$ cross section for the four-fermion final state and (ii) the invariant-mass distribution of $s \bar{s}$, in the case where $c \bar{c}$ are escaping detection and the corresponding integrated cross section $\left(\sigma_{2}\right)$. We will call a fermion "visible" if its transverse momentum is above $10 \mathrm{GeV}$ and $\left|\cos \theta_{\text {beam }}\right|<0.96$. Otherwise we call it "escaping detection". Our motivation for such a choice is the following: (i) it can be realized in practice by most detectors, (ii) it excludes jet-like activity in the initial state, such as off-mass-shell initial-state photon bremsstrahlung (or initial-state jet activity in the framework of the phenomenology of $p p$ colliders).

In general, we will follow the recommendations of LEP2 Workshop [4] for input parameters setting, but as it is essential for this paper, we will explain in detail all input parameters and switches of KORALW and grc4f in Appendices A and B.

As a centre-of-mass energy, we take 161,195 , and $350 \mathrm{GeV}$, corresponding respectively to $W$-pair production threshold, maximum expected centre-of-mass energy of LEP2, and $t \bar{t}$ threshold.

\subsection{General scheme of calculating uncertainty and numerical examples}

In the following we will present the general scheme for calculating the theoretical uncertainties of KORALW and grc4f MC predictions for observables in final states of the $W$-pair decay type. We will follow the general scheme that we have already applied in other cases $[3,12,13,14]$. For this purpose we need to collect numerical results obtained at different levels of approximation. This will enable us to calculate the size of different classes of corrections. Approximation levels are listed in the caption of the Tables 1,2 and the corresponding detailed information on the KORALW and grc4f input is given in Appendices A and B.

Let us start with the predictions at the lowest approximation level marked as input number ${ }^{3}$-2-, see Appendices A and B. In this case we use the constant width approximation, on mass-shell relation $\sin ^{2} \theta_{w}=1-M_{W}^{2} / M_{Z}^{2}$ and tree level doubly resonant $W$-pair production and decay diagrams (CC-03) only. The results for our

\footnotetext{
${ }^{3}$ Input number -1- corresponds to the case when the $W$ spin effects are switched off and is discussed later on.
} 
observables are collected for different centre-of-mass energies in Table 2a-c, entry -2-, and plotted in thick lines in Figs. 1-6.

Table 1: Total cross sections [pb] without any cuts for $c \bar{c} s \bar{s}$ production from KORALW and grc4f. Different input parameter settings for entries Nos. -2- to -5- are explained in Appendices A and B. The energies shown are: $161 \mathrm{GeV}(1 \mathrm{a}), 195 \mathrm{GeV}$ (1b) and $350 \mathrm{GeV}$ (1c). Please note numerical differences $(\sim 0.3 \%)$ due to different Coulomb correction implementation in KORALW and grc4f, and also due to e.g. YFS formfactor $\sim 0.7 \%$ which is switched on in KORALW.

$1 \mathrm{a}, \sigma_{t o t}[\mathrm{pb}]$, CMS energy $161 \mathrm{GeV}$.

\begin{tabular}{||c|c|c|c||}
\hline \hline No. & KORALW & grc4f & Comments \\
\hline 2. & $.5750 \pm .0002$ & $.5743 \pm .0007$ & CC-03 \\
3. & $.5676 \pm .0002$ & $.5660 \pm .0009$ & CC-03+leading loop corr. \\
4. & $.6271 \pm .0002$ & $.6273 \pm .0028$ & CC-43 \\
5. & $.6194 \pm .0002$ & $.6174 \pm .0006$ & CC-43+leading loop corr. \\
6. & $.4622 \pm .0002$ & $.4565 \pm .0005$ & CC-43+leading loop corr. + ISR \\
\hline \hline
\end{tabular}

1b, $\sigma_{\text {tot }}[\mathrm{pb}]$, CMS energy $195 \mathrm{GeV}$.

\begin{tabular}{||c|c|c|c||}
\hline \hline No. & KORALW & grc4f & Comments \\
\hline 2. & $2.2503 \pm .0007$ & $2.249 \pm .002$ & CC-03 \\
3. & $2.0981 \pm .0007$ & $2.095 \pm .002$ & CC-03+leading loop corr. \\
4. & $2.3274 \pm .0008$ & $2.323 \pm .003$ & CC-43 \\
5. & $2.1707 \pm .0007$ & $2.163 \pm .002$ & CC-43+leading loop corr. \\
6. & $1.9753 \pm .0009$ & $1.947 \pm .002$ & CC-43+leading loop corr. + ISR \\
\hline \hline
\end{tabular}

1c, $\sigma_{\text {tot }}[\mathrm{pb}]$, CMS energy $350 \mathrm{GeV}$.

\begin{tabular}{||c|c|c|c||}
\hline \hline No. & KORALW & grc4f & Comments \\
\hline 2. & $1.3771 \pm .0005$ & $1.377 \pm .002$ & CC-03 \\
3. & $1.2771 \pm .0005$ & $1.273 \pm .002$ & CC-03+leading loop corr. \\
4. & $1.4132 \pm .0006$ & $1.411 \pm .002$ & CC-43 \\
5. & $1.3101 \pm .0005$ & $1.304 \pm .001$ & CC-43+leading loop corr. \\
6. & $1.3616 \pm .0006$ & $1.344 \pm .001$ & CC-43+leading loop corr. + ISR \\
\hline \hline
\end{tabular}

Let us now proceed with different classes of corrections. First let us list those classes of higher order corrections that are expected to be enhanced with respect to $\frac{\alpha}{\pi} \sim 0.2 \%$ size. They include:

1. Coulomb interaction of the $W$-pair close to the production threshold,

2. running $Z$ and $W$ widths, 
Table 2: Total cross section $\sigma_{\text {tot }}[\mathrm{pb}]$ without cuts and cross sections $\sigma_{2}$ and $\sigma_{4}[\mathrm{pb}]$ of two or four "visible" fermions defined as in Section 2. Different input parameter settings as explained in Appendix $A$ and $B$ are used for entries No 1 to 6 . The energies shown are: $161 \mathrm{GeV}$ (2a), $195 \mathrm{GeV}$ (2b) and $350 \mathrm{GeV}$ (2c).

2a, CMS energy $161 \mathrm{GeV}$.

\begin{tabular}{||c|c|c|c|c||}
\hline \hline & $\sigma_{\text {tot }}$ & $\sigma_{2}$ & $\sigma_{4}$ & Comments \\
\hline 1 & $0.57519 \pm .0002$ & $.00041 \pm .00000$ & $0.50269 \pm .0002$ & CC-03 no spin \\
2 & $0.57504 \pm .0002$ & $.00035 \pm .00001$ & $0.50785 \pm .0002$ & CC-03 \\
3 & $0.56762 \pm .0002$ & $.00034 \pm .00001$ & $0.50152 \pm .0002$ & CC-03+lead. loop corr. \\
4 & $0.62714 \pm .0002$ & $.00885 \pm .00002$ & $0.52071 \pm .0002$ & CC-43 \\
5 & $0.61937 \pm .0002$ & $.00877 \pm .00002$ & $0.51429 \pm .0002$ & CC-43+lead. loop corr. \\
6 & $0.46219 \pm .0002$ & $.01022 \pm .00005$ & $0.36910 \pm .0002$ & Like 5 but with ISR \\
\hline \hline
\end{tabular}

2b, CMS energy $195 \mathrm{GeV}$.

\begin{tabular}{||c|c|c|c|c||}
\hline \hline & $\sigma_{\text {tot }}$ & $\sigma_{2}$ & $\sigma_{4}$ & Comments \\
\hline 1 & $2.25006 \pm .0006$ & $.00480 \pm .00003$ & $1.83470 \pm .0006$ & CC-03 no spin \\
2 & $2.25028 \pm .0007$ & $.00382 \pm .00003$ & $1.85607 \pm .0007$ & CC-03 \\
3 & $2.09812 \pm .0007$ & $.00359 \pm .00003$ & $1.72991 \pm .0006$ & CC-03+lead. loop corr. \\
4 & $2.32744 \pm .0008$ & $.00989 \pm .00004$ & $1.90338 \pm .0008$ & CC-43 \\
5 & $2.17071 \pm .0007$ & $.00950 \pm .00004$ & $1.77361 \pm .0007$ & CC-43+lead. loop corr. \\
6 & $1.97533 \pm .0009$ & $.01022 \pm .00008$ & $1.61557 \pm .0008$ & Like 5 but with ISR \\
\hline \hline
\end{tabular}

2c, CMS energy $350 \mathrm{GeV}$.

\begin{tabular}{||c|c|c|c|c||}
\hline \hline & $\sigma_{\text {tot }}$ & $\sigma_{2}$ & $\sigma_{4}$ & Comments \\
\hline 1 & $1.37670 \pm .0004$ & $.03055 \pm .00007$ & $0.76319 \pm .0003$ & CC-03 no spin \\
2 & $1.37708 \pm .0005$ & $.00713 \pm .00004$ & $0.77531 \pm .0004$ & CC-03 \\
3 & $1.27712 \pm .0005$ & $.00662 \pm .00004$ & $0.71982 \pm .0004$ & CC-03+lead. loop corr. \\
4 & $1.41318 \pm .0005$ & $.00913 \pm .00005$ & $0.79474 \pm .0004$ & CC-43 \\
5 & $1.31010 \pm .0005$ & $.00853 \pm .00005$ & $0.73714 \pm .0004$ & CC-43+lead. loop corr. \\
6 & $1.36161 \pm .0006$ & $.00871 \pm .00005$ & $0.79493 \pm .0005$ & Like 5 but with ISR \\
\hline \hline
\end{tabular}

3. effective coupling constants which affect the relation: $\sin ^{2} \theta_{w}=1-M_{W}^{2} / M_{Z}^{2}$,

4. YFS formfactor and QED Initial-State Radiation (ISR) for the KORALW or QEDPS [15] for the grc4f.

It is rather straightforward to include first three types of corrections in the case of CC-03 amplitudes and the appropriate results are collected in Table $2 \mathrm{a}-\mathrm{c}$ under entry -3-. In this case we follow a recommendation of the LEP2 Workshop [4] for so-called CC-03 diagrams in the choice of definition of $\sin ^{2} \theta_{W}$ (see Appendix A for details) and in switching to $s$-dependent $Z$ and $W$ widths. As we can see, corrections are typically rather small. We thus expect higher-order corrections to be negligible. 
Let us also point out that the dominant higher order corrections of the vacuum polarization type are already included in definitions of running widths and effective coupling constants. The systematic uncertainty related to those corrections is thus included in the uncertainty on the numerical values of the program input parameters and not separable from them.

The Coulomb correction implemented in KORALW is taken from Refs. [16, 17], whereas grc4f takes it from Ref. [18]. This leads to numerical results that differ by about $0.3-0.4 \%$ for all energies. According to [4] (vol. 1, p. 117-119) the deviating formulae of Refs. [16] and [18] constitute equally well justified representations of the Coulomb phenomenon; however, see also Ref. [16] for a detailed discussion. Nonetheless this $0.3-0.4 \%$ difference will be reflected (indirectly) in our final estimate of physical precision. As for the higher-order corrections beyond $\mathcal{O}(\alpha)$ to Coulomb correction, it is shown (see e.g. $[17,4]$ ) that they are numerically small, below $0.1 \%$ and can thus be neglected in our considerations.

Let us now turn to the effects of complete tree level amplitudes. To this end we have to switch off all corrections introduced in entry -3- and return to the input definition as in entry -2-; we then implement the complete set of tree level spin amplitudes, the so-called CC-43 graphs. We collect results under entry -4- of Tables 1 and 2 and in the thin line of Figs. 2, 4 and 6. As we can see, the cross-section increases by a few per cent. This is mainly due to the inclusion of the $Z-\gamma$ intermediate state. Note that when complete Born level spin amplitudes are included, i.e. in the presence of a $Z$ peak, another, $Z$-like peak, in $s \bar{s}$ invariant-mass distribution at higher centre-of-mass energy, looks particularly suggestive, see Figs. 2, 4 and 6. The cross section for this faked resonance is $0.0015 \mathrm{pb}$ at $195 \mathrm{GeV}$, which translates to about 2 or 3 events per overall time of operation of every LEP experiment if the summation over all hadronic final states is performed. (We will return to this phenomenon in Sect. 3).

The simultaneous inclusion of the complete set of tree-level diagrams and incomplete $\mathcal{O}(\alpha)$ corrections, for example running $W$-width, as in entry -3-, breaks the gauge invariance. See e.g. [19] and also [20, 21, 22]. The other corrections of entry -3- may also cause numerical effects upon combining 'by hand' with background graphs. Until a complete $\mathcal{O}(\alpha)$ calculation for the four-fermion processes is done, the question of coherent extension of partial $\mathcal{O}(\alpha)$ corrections to the background case will always be present.

For the time being we proceed as follows. Formally speaking, in order to combine corrections of entries -3- and -4- of our Tables 1 and 2 it is necessary to perform several runs of the $\mathrm{MC}$ and later to add the corresponding corrections according to the following formula:

$$
X=X_{2}+\left(X_{3}-X_{2}\right)+\left(X_{4}-X_{2}\right)+\text { h.o.t. }
$$

where $X_{i}$ correspond to the different approximations (of observable $X$ ) as summarized in the caption of Tables 1 and 2. Equation (1) is nothing more than the multiparameter linear interpolation based on Taylor expansion in some "parameter 
space' (symbolically denoted by $f$ 's), in which each direction corresponds to a given set of 'orthogonal' corrections (-3- and -4- for example):

$$
\Delta X\left(f_{3}, f_{4}\right)=\frac{\partial X}{\partial f_{3}} \Delta f_{3}+\frac{\partial X}{\partial f_{4}} \Delta f_{4}+\cdots \equiv \Delta X_{3}+\Delta X_{4}+\text { h.o.t. }
$$

and the missing terms are of higher order in the expansion. Approximation (1) therefore gives a safe way of combining corrections.

On the other hand this solution may be rather painstaking, as in the case of an observable including complicated experimental cuts, several simulation runs for the same processes but different theoretical input may be necessary. This may take an enormous amount of CPU time if the full detector simulation is requested.

For this practical reason we propose a different way of combining corrections. It simply amounts to switching all of them simultaneously. This common sense interpolation is of course Monte Carlo dependent, as each code most likely uses different ad hoc method of combining corrections. The ultimate test of common sense interpolation can be done by comparison with the linear interpolation of eq. (1). If these two interpolations agree within the required precision, then one can safely use common sense interpolation. However, this has to be checked for each type of observable separately, just as we do for $s \bar{s} c \bar{c}$ ones in here. Specifically, in entry -5- of Table 2 we give the result of simultaneous switching on corrections -3and -4-. We can see by direct inspection of Table 2 that our eq. (1) reproduces at $0.3 \%$ precision level entry -5 - from the corresponding entries -2- and -4-. Strictly speaking, this is true for total and $\sigma_{4}$ cross sections. For $\sigma_{2}$ the agreement is at the $1 \%$ level. However, as the $\sigma_{2}$ is typically two orders of magnitude smaller than the others, the $1 \%$ agreement is more than sufficient. We can therefore conclude that, in this case, our common sense interpolation approach of simultaneous inclusion of all corrections works at the required precision level.

So far, our results were obtained with ISR and YFS form factors switched off. If we add these effects, we obtain finally predictions including most of the necessary corrections. ${ }^{4}$ We collect results in entries -6 - of Table 2.

This way, we have exhausted our list of $\mathcal{O}(\alpha)$ enhanced corrections, and we are left with the remaining, genuine $\mathcal{O}\left(\frac{\alpha}{\pi}\right)$ ones. To state it simply, before a complete $\mathcal{O}(\alpha)$ calculation of corrections to $e^{+} e^{-} \rightarrow 4 f$ are available, the general uncertainty of the $\mathcal{O}\left(\frac{\alpha}{\pi}\right)$, due to $\mathrm{QED} / \mathrm{EW}$ corrections, must be assumed in the results.

At this moment in order to quantify the above vague statement we will use a number of partial results on $\mathcal{O}\left(\frac{\alpha}{\pi}\right)$ corrections. We will briefly cover them in the following.

\footnotetext{
${ }^{4} \mathrm{~A}$ brief discussion of QED initial-state corrections was presented already at the LEP2 Workshop. One has to keep in mind that even though kinematical configurations with an arbitrary number of photons are generated by our programs and corresponding uncertainties were discussed for KORALW in Ref. [6], and for grc4f in Ref. [23], matrix element is limited to the second-order leading-logarithmic accuracy in both cases (on top of correct all-order soft limit!).
} 
1. We briefly reviw the formulation of QEDPS that is used in the grc4f. The algorithm is completely in parallel with that of the parton-shower model in perturbative QCD, which has been well known for a long time. The basic assumption is that the structure function of an electron, with the virtuality $Q^{2}$ and the momentum fraction $x$, obeys the Altarelli-Parisi equation

$$
\frac{d D\left(x, Q^{2}\right)}{d \ln Q^{2}}=\frac{\alpha}{2 \pi} \int_{x}^{1} \frac{d y}{y} P_{+}(x / y) D\left(y, Q^{2}\right)
$$

in the leading-log (LL) approximation [24]. This equation can be converted to the integral equation

$D\left(x, Q^{2}\right)=\Pi\left(Q^{2}, Q_{s}^{2}\right) D\left(x, Q_{s}^{2}\right)+\frac{\alpha}{2 \pi} \int_{Q_{s}^{2}}^{Q^{2}} \frac{d K^{2}}{K^{2}} \Pi\left(Q^{2}, K^{2}\right) \int_{x}^{1-\epsilon} \frac{d y}{y} P(y) D\left(x / y, K^{2}\right)$,

where $\Pi$ is the Sudakov factor. Here, rigorously speaking, $Q_{s}^{2}$ should be $m_{e}^{2}$ as it gives the initial condition. For simplicity the fine structure constant $\alpha$ is assumed not running with $Q^{2}$.

The integral form eq. (4) can be solved by iterating the right-hand side in a successive way. Then it is apparent that the emission of $n$ photons corresponds to the $n$-th iteration. The information on the transverse momentum can be obtained by solving the kinematical equation.

In the formulation there appear two parameters, $Q_{s}^{2}$ and $Q_{0}^{2}$. In the program the following values are chosen:

$$
Q_{s}^{2}=m_{e}^{2} e=m_{e}^{2} \times 2.71828 \cdots, \quad Q_{0}^{2}=10^{-12} \mathrm{GeV}^{2}
$$

The former value was settled to effectively take into account the constant term -1 of $\beta$ in such a way that $\beta=(2 \alpha / \pi)\left(\ln \left(s / m_{e}^{2}\right)-1\right)=(2 \alpha / \pi) \ln \left(s /\left(m_{e}^{2} e\right)\right)$. Since the second parameter is unphysical, any physical observable should not depend on it. It has been checked that increasing $Q_{0}^{2}$ up to $O\left(m_{e}^{2} / 10\right)$ leaves the result unchanged in the statistical error of the event generation.

We conclude that, in total, the related uncertainty is (a) $0.1 \%$ from comparison between the QEDPS and the structure function method and (b) $0.65 \%$ due to neglecting the overall $K$ factor from the exact $O(\alpha)$ calculation[23].

2. Let us now go back to the YFS scheme and to the discussion of the main uncertainty (in that language) related to initial-state bremsstrahlung. The YFS formfactor is a consequence of the rigorous and general program of resummation of soft photons in any QED process, as given in the classical paper [25]. In that sense it is universal and process-independent. On the other hand, it originates from a particular (although well motivated) choice of approximation in extracting 'soft' parts from the Feynman graphs. It can thus be argued 
that there is some arbitrariness in its definition. In $s$-channel reactions such as muon or tau pair production, this formfactor, which numerically constitutes $+0.7 \%$ overall correction, had to be included. This was based on the direct calculation of QED corrections up to the second order in $\alpha$. In the case of $W$-pair production such calculations are missing so far, and there are opinions [26] that such correction (YFS formfactor) should perhaps not be included because in this case cancellation of terms $\sim \frac{\alpha}{\pi} \cdot \pi^{2}$ from different sources cannot be understood.

3. In Ref. [27], the YFS resummation of real and virtual soft photons is extended to the case of bremsstrahlung from heavy bosons $W$. This is done for massive $W$ 's with finite width in a manifestly gauge-invariant way. The results of the calculation are then implemented in the four-fermion Monte Carlo program YFSWW2. The numerical results presented in Ref. [27] show that, for the total cross section, the net effect of real+virtual emission from $W$-pair, after subtracting the Coulomb correction, is of the size of $0.4 \%$ for LEP2 energies and $0.8 \%$ at $500 \mathrm{GeV}$. The size of this correction, absent in KORALW, should be included in the final physical uncertainty.

4. Another technique of computing ISR in a gauge-invariant way has been developed in [28]. The $t$-channel neutrino was split into two oppositely flowing charges. These charges were then ascribed to initial and intermediate ( $W$ 's) states respectively. In this way, certain terms effectively have been rearranged between initial- and intermediate-state-type corrections. Numerical results presented in [28] are: $0.4 \%$ for LEP2 energies and $1.5 \%$ for $500 \mathrm{GeV}$. This is in qualitative agreement with the results of Ref. [27].

5. The interference effects to the process of $W$-pair production and decay have been analysed in $[29,30]$. It has been shown therein that, for sufficiently inclusive quantities (e.g. total cross section) these effects, both real and virtual, are suppressed by an additional $\Gamma_{W} / M_{W}$ factor with respect to the genuine $\frac{\alpha}{\pi}$ corrections.

6. Finally, another possible way of estimating QED/EW uncertainties is to use the results of the calculation of the complete $\mathcal{O}(\alpha)$ corrections to stable onshell $W$-pair production of Refs. [31, 32]. These corrections are, however, to our knowledge, not implemented in a full-scale four-fermion Monte Carlo program, which makes direct comparisons more difficult. Based on these onshell results the uncertainty of the four-fermion total cross-section at LEP 2 has been estimated in Ref. [4] (vol. 1, p. 127) to be $2 \%$ for $161 \mathrm{GeV}, 1-2 \%$ for 175 and $190 \mathrm{GeV}$.

In the following we will mostly rely in our estimates of the QED/EW uncertainty on the results of Ref. [27]. Specifically we take as the estimate the size of the YFS form factor for the ISR, summed linearly, as discussed above with the size 
of intermediate state ( $W^{\prime}$ 's) YFS real+virtual corrections of [27]. On top of that we impose a safety factor of 2 . This procedure yields $2 \times(0.7 \%+0.4 \%) \simeq 2 \%$ and we quote a total QED/EW uncertainty of $2 \%$. (The study of the QEDPS also gives $0.7 \%$ uncertainty from comparison between the QEDPS and the exact $O(\alpha)$ calculation.) Let us note also, that we did not explicitly include the $0.4 \%$ uncertainty due to Coulomb correction discrepancy between Refs. [16] and [18] mentioned earlier. We include it in the safety factor of 2 (it is in a sense related to the $W$-state uncertainty). ${ }^{5}$. This is not an improvement with respect to the similar precision estimate for the total cross section based on on-shell experience $(2 \%$ for $161 \mathrm{GeV}$ and $1-2 \%$ for 175 and $190 \mathrm{GeV}$ ) given in Ref. [4] (vol. 1, p. 127).

We hope that a careful study of terms proportional to $\frac{\alpha}{\pi} \cdot \pi^{2}$ with special emphasis on the matching terms from different calculations of partial $\mathcal{O}(\alpha)$ contributions may lead to a reduction of this systematic error. However, the final answer may have to wait for results of complete $\mathcal{O}(\alpha)$ calculation.

Another uncertainty, related to higher-order parts of our ansatz on initial state bremsstrahlung matrix element, we take as $0.1 \%$ and discard. On the basis of inspection of our Tables, we believe that our ansatz on the simultaneous inclusion of all corrections even though it violates in principle gauge invariance, in practice introduces for our observables an uncertainty of order of $0.1 \%$ which we discard as well.

The other component of the total uncertainty to be mentioned here is the question (briefly covered in the introduction) of the technical precision of KORALW and grc4f codes. For KORALW it was discussed already in [6] and for grc4f in [4]. Numerical results of the present paper were reproduced by the two programs and agreement was found at the level of $0.3 \%$. Based on all these factors, we conclude that in the case of our observables the technical precision of both the KORALW and grc4f programs is not worse than $0.3 \%$, and therefore negligible in comparison to the physical one.

We believe that we can sum all the above uncertainties in quadrature, obtaining a final result for the uncertainty of KORALW/grc4f predictions, including technical and electroweak effects, for our observables of $2 \%$. The dominant source of uncertainty originates from the lack of complete $\mathcal{O}(\alpha)$ calculation of electroweak corrections to combined $W$-pair production and decay process.

We have excluded from our considerations an uncertainty in the program numerical input parameters, such as the $Z$ and $W$ masses and widths as well as the $W$ branching ratios. Note that in $W$-width and $W$ branching ratios there are hidden total final-state QCD corrections. The related uncertainty is not included here either.

We have excluded from our discussion yet another source of systematic error which is related to hadronization, jet definition, QCD perturbative effects in the final state, etc. Note that this includes final-state QED bremsstrahlung from quarks

\footnotetext{
${ }^{5}$ However a direct summation of all three uncertainties gives $1.5 \%$ bottom-rock precision level.
} 
which cannot be separated from jet formation. These effects may constitute sizeable corrections, especially at higher energies. For the purpose of simulation of such effects we use the JETSET algorithm and we refer to [4] for a discussion of therelated uncertainty. As suggested in [4] (vol. 2, p. 172; "good bet" approach) we leave this topic to independent research. We stop at generating a random choice for colour recombination between charged-current (CC) and neutral-current (NC) configurations according to the size of corresponding separate matrix elements squared. So far, we neglect interference effects between the two configurations, as well as related Bose-Einstein effects. For more details, see Ref. [4] vol. 1 p. 124 and 190. We believe, however, that effects and uncertainties related to QCD/hadronization may require additional separate studies to be completed in the near future [33].

\section{Spin correlations}

In this section we will show the importance of spin correlations in the presence of cut-offs in $W$-pair production on the example of $e^{+} e^{-} \rightarrow s \bar{s} c \bar{c}$. This is to be contrasted with the 'no correlations' case of $e^{+} e^{-} \rightarrow W^{+} W^{-} \rightarrow s \bar{s} c \bar{c}$ where $W$ 's are produced 'on shell' and then independently decayed. To this end we return to the 'lowest approximation level', as described in Sect. 2.1, marked as entry -2- in Table 2 and shown in thick lines in Figs. 1, 3 and 5. We now switch the spin correlations off. The results for the same observables and cut-offs as before are shown in Figs. 1, 3 and 5 (thin lines) and in Table 2 (input number -1- ).

Let us point again to the spectacular peak in the $s \bar{s}$ invariant-mass distribution, which becomes more and more profound for centre-of-mass energies higher above the $W W$ threshold. It is present in the case -2- (thick line) but not (!) in the case -1- (thin line) when spin correlations are switched off. It is therefore a genuine effect of an interplay of our veto cut-off on $c$-quarks with spin correlations in $W$ decays! This exercise proves that any kind of 'on-shell' approximation of this kind may lead not only to quantitative few or several per cent inaccuracies, but, upon applying cut-offs, to misleading qualitative changes in the overall picture.

Note that the cross cection for our faked 'object' is of the order of $0.0015 \mathrm{pb}$ for the $c \bar{c} s \bar{s}$ final state alone. This translates to 2 or 3 such events per LEP collaboration if all hadronic final states are taken into account.

Moreover, it should be kept in mind that our choice of observables and cut-offs is in a sense random and that similar effects can be expected in other distributions as well.

\section{Summary}

In this paper we have discussed different types of non-hadronization corrections for the three observables: 'visible four-quark' total cross section and invariant mass distribution and cross section of 'visible two quarks' from the $s \bar{s} c \bar{c}$ final state at 
LEP2 and higher energies. We have found that in most cases MC simulation can incorporate all necessary corrections, such as Coulomb corrections, effective coupling constants, running $Z$ and $W$ width and complete set of all tree-level diagrams. Even if in principle it can be shown that such a solution leads to gauge non conservation, we have found that for practical purposes, as in the cases presented above, all corrections can be included simultaneously. This common sense interpolation works because of our reasonable/lucky choice of the gauge and observables discussed in this paper. This is of practical importance as it eliminates the need to calculate corrections separately in several runs of detector simulation.

We conclude that the overall uncertainty of theoretical predictions for our observables is $2 \%$ with dominating contribution from the lack of complete $\mathcal{O}(\alpha)$ calculations of combined $W$-pair production and decay process. This is already a factor of 4 short of the ultimate goal for LEP2 phenomenological preparations, even though we still exclude further $Q C D /$ hadronization uncertainties. It is, however, sufficiently precise for the statistics of the first year of LEP2 operation.

On the other hand, we have found that seemingly innocent and natural choices of cuts (which can be motivated by detector or background elimination needs) may lead to a very strong deformation of the 4-fermion signal and even to unexpected faked peaks strongly enhanced by spin effects. We expect that a careful study of $\mathrm{MC}$ predictions, including complete spin effects, may be necessary in many cases such as four-jet/leptons final states or six-jet/lepton final states (e.g. involved in $t \bar{t}$ production and decay). This is important not only in the case when a given channel is studied by itself, but also if it is treated as the background.

Two of the authors(T.I., Y.K.) wish to thank our colleagues in the MinamiTateya collaboration, J. Fujimoto, T. Kaneko, K. Kato, S. Kawabata, T. Munehisa, N. Nakazawa, D. Perret-Gallix, Y. Shimizu and H. Tanaka. They are supported in part by the Grant-in-Aid(No.07044097) of Monbu-sho, Japan. M.S. and Z.W. are

pleased to thank S. Jadach, W. Płaczek and B.F.L. Ward for numerous discussions and other kinds of support in this study.

\section{References}

[1] G. Altarelli, R. Kleiss and C. Verzegnassi, eds., Z PHYSICS at LEP 1, 3 vols., CERN, Geneva, 1989.

[2] M. Grünewald, Humboldt Universität Berlin, preprint HUB-IEP-95/13 (unpublished).

[3] S. Jadach, E. Richter-Wạs, B. F. L. Ward, and Z. Wạs, Phys. Lett. B353, 362 (1995), CERN preprint CERN-TH/95-38. 
[4] G. Altarelli, T. Sjöstrand and F. Zwirner, eds., Physics at LEP 2, 2 vols., CERN 96-01, Geneva, 1996.

[5] M. Skrzypek, S. Jadach, W. Płaczek, and Z. Wạs, Comput. Phys. Commun. 94 (1996) 216.

[6] M. Skrzypek et al., Phys. Lett. B372 (1996) 289.

[7] J. Fujimoto, T. Ishikawa, T. Kaneko, K. Kato, S. Kawabata, Y. Kurihara, T. Munehisa, D. Perret-Gallix, Y. Shimizu, H. Tanaka, , 'grc4f v1.1: A fourfermion event generator for $e^{+} e^{-}$collisions', to appear in Comput. Phys. Commun.

[8] J. Fujimoto, T. Ishikawa, T. Kaneko, K. Kato, S. Kawabata, Y. Kurihara, T. Munehisa, N. Nakazawa, Y. Shimizu, H. Tanaka, GRACE User's manual, version 2.0, Minami-Taneya collaboration, submitted to Comput. Phys. Commun.

[9] S. Kawabata, Comput. Phys. Commun. 41 (1986) 127, and 88 (1995) 309.

[10] E.E. Boos, M.N. Dubinin, V.A. Ilyin, A.E. Pukhov, S.A. Shichanin, T. Kaneko, S. Kawabata, Y. Kurihara, Y. Shimizu and H. Tanaka, Int. J. Mod. Phys. C5 615 (1994).

[11] E. Boos et al., in: '91 Electroweak Interactions and Unified Theories (Proc. of the XXVIth Rencontre de Moriond), J.Tran Thanh Van, ed. (Editions Frontiéres, 1991), p. 501.

E. Boos et al., in: New Computing Techniques in Physics Research II (Proc. of the Second Int.Workshop on Software Engineering, Artificial Intelligence and Expert Systems in High Energy and Nuclear Physics), D. Perret-Gallix, ed. (World Scientific, 1992), p. 665.

E. Boos et al., SNUTP preprint 94-116, Seoul, 1994 (hep-ph/9503280)

[12] F. Sanchez and Z. Wạs, Phys. Lett. B351 (1995) 562.

[13] S. Jadach, B. Ward, and Z. Wạs, Phys. Lett. B257 (1991) 213.

[14] P. Colas, R. Miquel, and Z. Wạs, Phys. Lett. B246 (1990) 541.

[15] J. Fujimoto, Y. Shimizu and T. Munehisa, Prog. Theor. Phys. 90 (1993) 177, and 91 (1994) 333.

[16] V. S. Fadin, V. A. Khoze, A. D. Martin and A. Chapovsky, Phys. Rev. D52 (1995) 1377.

[17] V. S. Fadin, V. A. Khoze, A. D. Martin and W. J. Stirling, Phys. Lett. B363 (1995) 112. 
[18] D. Bardin, W. Beenakker, A. Denner Phys. Lett. B317 (1993) 213.

[19] Y. Kurihara, D. Perret-Gallix, and Y. Shimizu, Phys. Lett. B349, (1995) 367.

[20] U. Baur, D. Zeppenfeld, Phys. Rev. Lett. 75 (1995) 1002.

[21] E. Argyres et.al., Phys. Lett. B358 (1995) 339.

[22] W. Beenakker et al., preprint NIKHEF 96-031, PSI-PR-96-41, December 1996.

[23] T. Munehisa, J. Fujimoto, Y. Kurihara and Y. Shimizu, KEK CP-034, KEK Preprint 95-114, 1995, to appear in Prog. Theor. Phys.

Y. Kurihara, J. Fujimoto, T. Munehisa and Y. Shimizu, KEK CP-035, KEK Preprint 95-126, 1995, to appear in Prog. Theor. Phys.

[24] R. Odorico, Nucl. Phys. B172 (1980) 157, G. Marchesini and B.R. Webber, Nucl. Phys. B238 (1984) 1.

[25] D.R. Yennie, S. Frautschi and H. Suura, Ann. Phys. 13 (1961) 379.

[26] R. Kleiss, private communication.

[27] S. Jadach, W. Płaczek, M. Skrzypek and B.F.L. Ward, Phys. Rev. D54 (1996) 5434.

[28] D. Bardin, M. Bilenkii, A. Olchevski, and T. Riemann, DESY-93-035-REV, Jul 1993, Revised version, hep-ph/9507277.

[29] V.S. Fadin, V.A. Khoze and A.D. Martin, Phys. Rev. D49 (1994) 2247.

[30] V.S. Fadin, V.A. Khoze and A.D. Martin, Phys. Lett. B320 (1994) 141.

[31] J. Fleischer, F. Jegerlehner and M. Zralek, Z. Phys. C42 (1989) 409;

K. Kolodziej and M. Zralek, Phys. Rev. D43 (1991) 3619;

J. Fleischer, F. Jegerlehner and K. Kolodziej, Phys. Rev. D47 (1993) 830.

[32] M. Böhm et al., Nucl. Phys. B304 (1988) 463;

W. Beenakker, K. Kolodziej and T. Sack, Phys. Lett. B258 (1991) 469;

W. Beenakker, F.A. Berends and T. Sack, Nucl. Phys. B367 (1991) 287.

[33] S. Jadach et. al., in preparation. 


\section{Appendix A}

In this appendix we carefully review input parameters as used in the production of our Tables with the help of KORALW.

1. $* \operatorname{set} 1 *$

$* * * * * * * * * *$

We will start with parameters as set for entry -1- of our Tables and later on we will concentrate on respective differences only. The exact meaning of the parameters is described in the KORALW documentation, see Ref. [5].

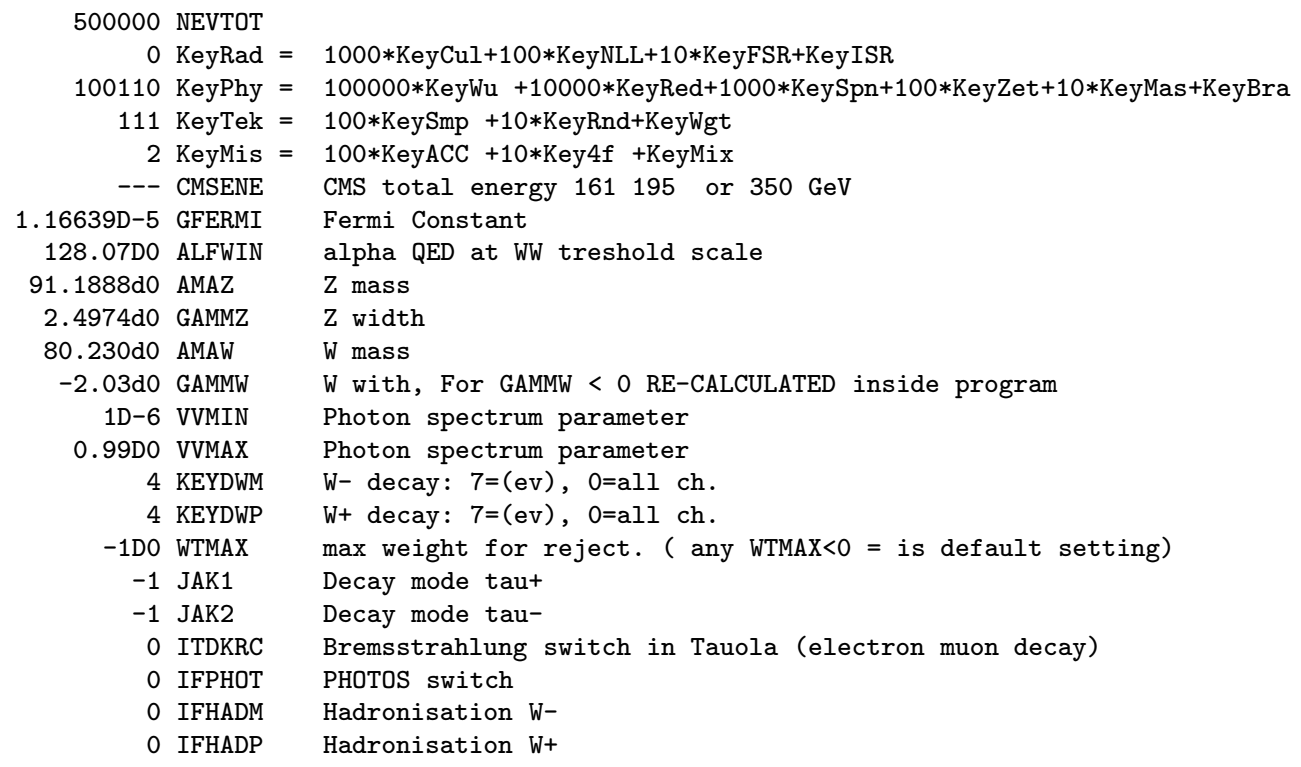

Along with the above parameters, there are other parameters set in routine FILEXP; see short extract from the KORALW printout below

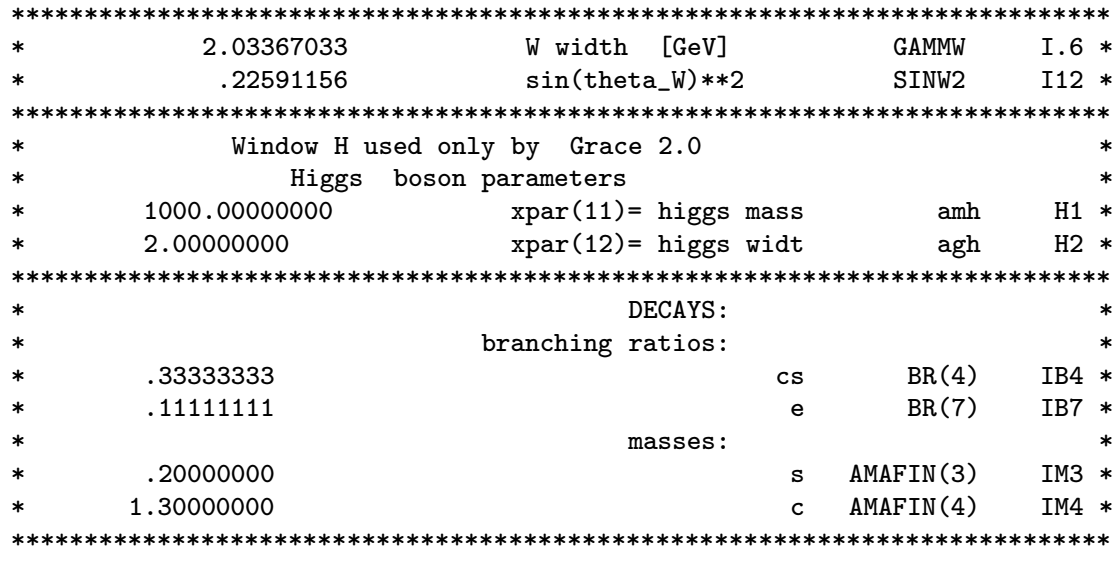


Please note that we have used here constant $Z$ and $W$ widths as well as $\sin ^{2} \theta_{W}=1-\frac{M_{W}^{2}}{M_{Z}^{2}} ;$ KeyWu=KeyZet=1; KeyMix=2. In our approach we could include QCD corrections and $C K M$ mixing as explained in [4], vol. 1 p. 104. This translates simply to a change of numerical input to KORALW in setting the $W$ total width and $W$ decay branching ratios to $e \nu$ and $c s$. We exclude, however, this correction from our considerations.

2 . $*$ set 2 (modifications with respect to set 1) * ****************************************************

Let us now continue with the list of corrections only. To switch spin correlations on, we leave all parameters as in previous run except:

101110 KeyPhy $=100000 *$ KeyWu $+10000 *$ KeyRed $+1000 *$ KeySpn $+100 *$ KeyZet $+10 *$ KeyMas + KeyBra

3. $*$ set 3 (modifications with respect to set 2) *

$* * * * * * * * * * * * * * * * * * * * * * * * * * * * * * * * * * * * * * * * * * * * * * * * * * *$

Next, we switch on the Coulomb interaction, ${ }^{6}$ KeyRad=1000, running $Z$ and $W$ width KeyPhy=1010 and change the definition of $\sin ^{2} \theta_{w}$. The KeyMis $=0$ activates a different mode of calculating $\sin ^{2} \theta_{w}$ as advocated in [4]

$$
\sin ^{2} \theta_{w}=\frac{\pi \alpha\left(M_{W}\right)}{\sqrt{2} M_{W}^{2} G_{\mu}} .
$$

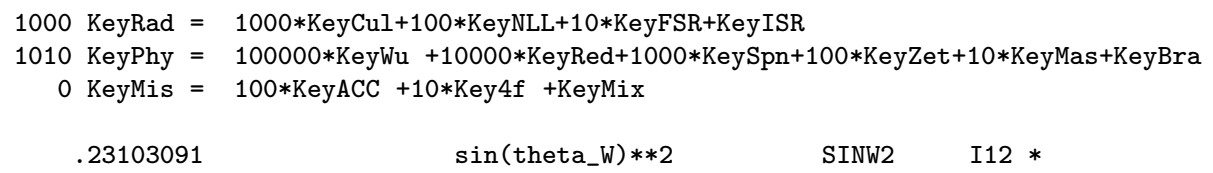

Note that in this case we use for the propagators:

$$
\frac{1}{s-M_{X}^{2}+\theta(s) \Gamma_{X} s / M_{X}}
$$

\section{4. * set 4 (modifications with respect to set 2) *} ****************************************************

Here we switch on all tree-level spin amplitudes, but we switch off all corrections introduced in set 3 above. In comparison to set 2 , we introduce the following modifications:

12 KeyMis $=100 *$ KeyACC $+10 *$ Key4f + KeyMix

\footnotetext{
${ }^{6}$ Note that QED coupling is taken at the low-energy limit alfinv= $137.036 \ldots$
} 
5. $*$ set 5 (modifications with respect to set 3 ) *

****************************************************

Now we turn to the combined case with all tree-level spin amplitudes, running widths and Coulomb correction. Also the $\sin ^{2} \theta_{w}$ is calculated by the program in the same way as in the case of set 3 :

10 KeyMis $=100 *$ KeyACC $+10 *$ Key $4 \mathrm{f}+\mathrm{KeyMix}$

6. $*$ set 6 (modifications with respect to set 5 ) *

$* * * * * * * * * * * * * * * * * * * * * * * * * * * * * * * * * * * * * * * * * * * * * * * *$

Finally with the last case we switch on the initial state bremsstrahlung and YFS form factor:

1101 KeyRad $=1000 *$ KeyCul $+100 *$ KeyNLL $+10 *$ KeyFSR+KeyISR

\section{Appendix B}

In this Appendix we show those input parameters used in the production of our Tables with the help of grc4f. They are printed on the first page of an output as an 'echo-back', as shown below. This is for the case set 4. Description of these input parameters for each set will be given later. In grc4f, however, we do not prepare the process for the set 1.

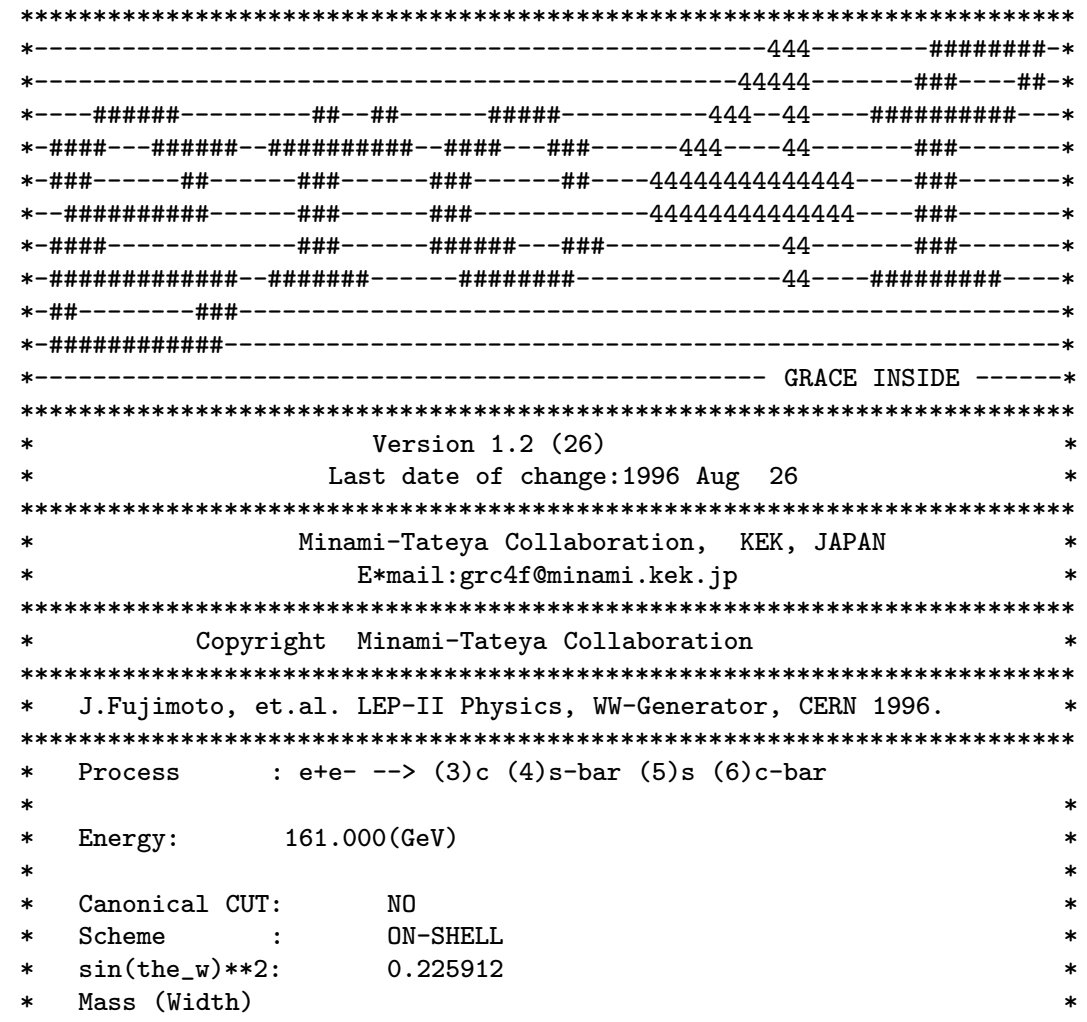




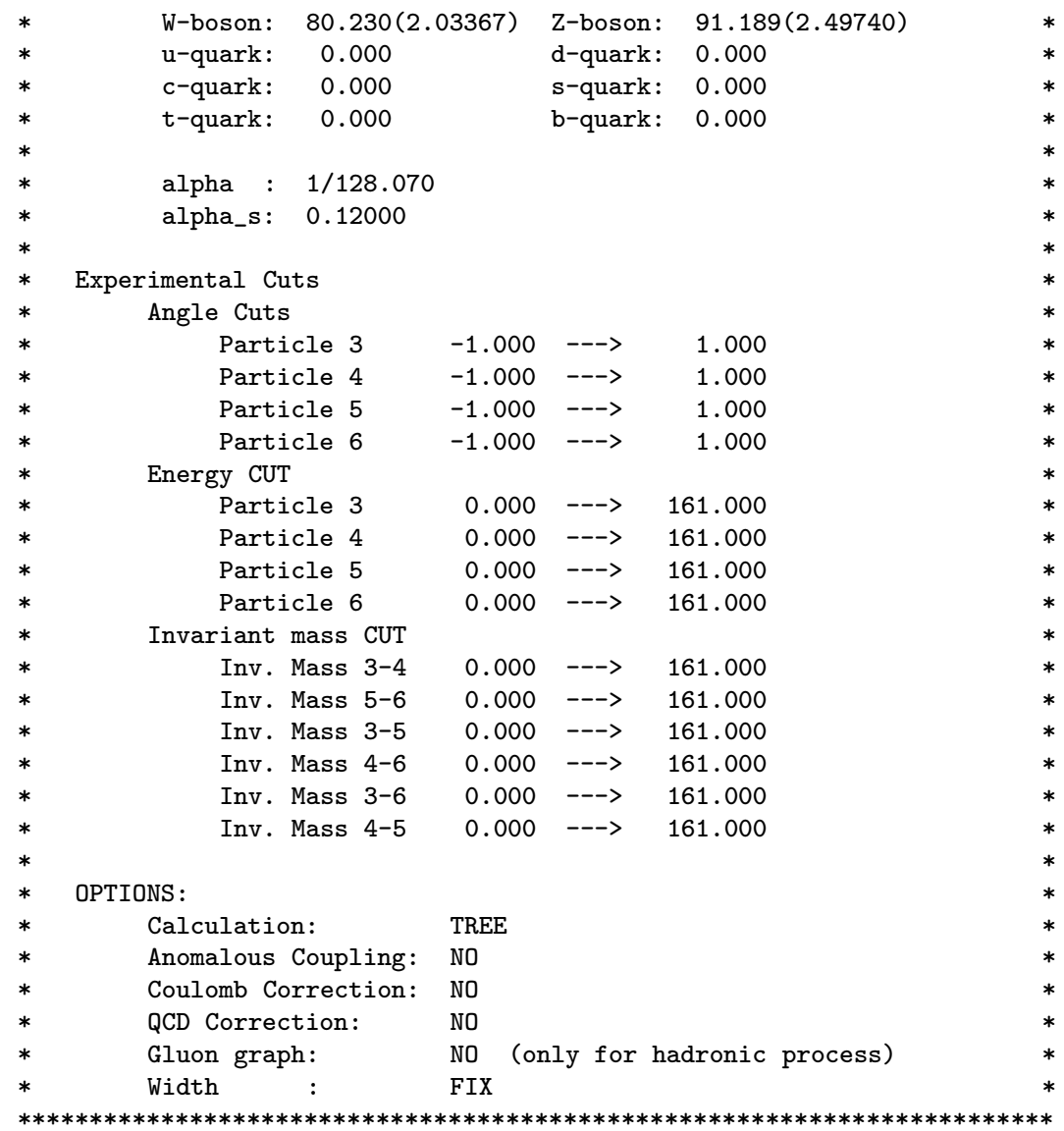

\section{1. $*$ set $4 *$}

$* * * * * * * * * *$

We start with parameters as the set for entry 4 of our Tables and then we indicate respective differences only. The exact meaning of the parameters is described in the grc4f documentation, see Ref. [7].

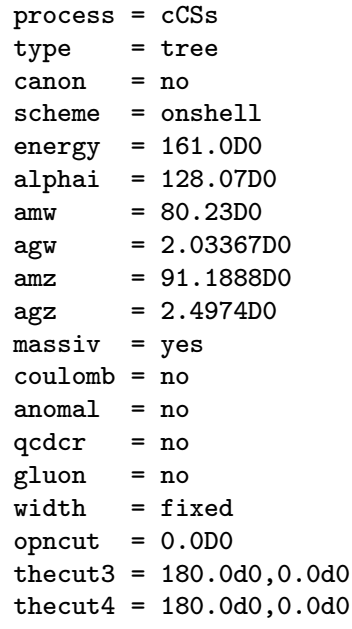




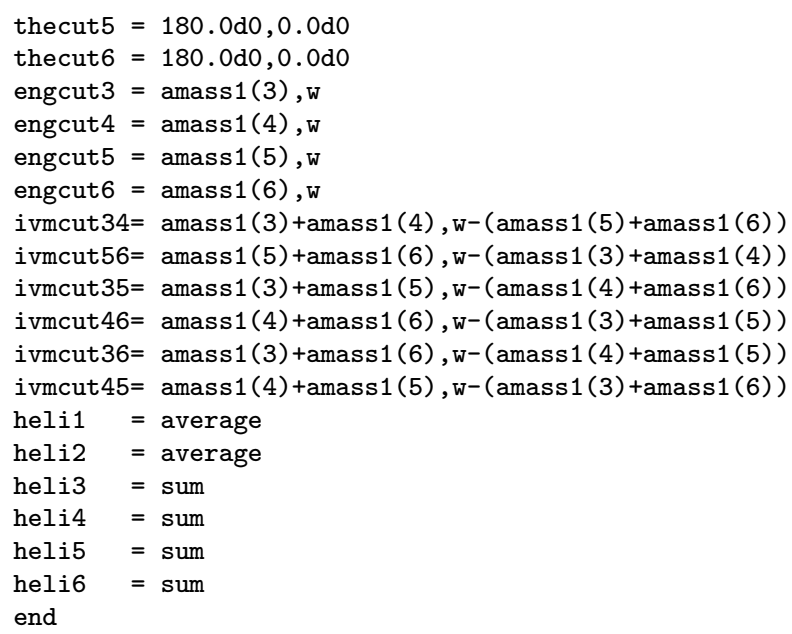

2. $*$ set 5 (modifications with respect to set 4 ) * ***************************************************

We introduce the following modifications to set 4 .

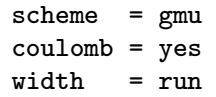

The output is changed:

* Scheme : $\quad$ G_mu

* $\quad \sin ($ the_w $) * * 2: \quad 0.231031$

* Coulomb Correction: YES

* Width $\quad$ : $\quad$ RUN

\section{3. * set 6 (modifications with respect to set 5)*} $* * * * * * * * * * * * * * * * * * * * * * * * * * * * * * * * * * * * * * * * * * * * * * * *$

We introduce the following modifications to set 5 .

type $=$ qedpsi

The output becomes:

Calculation: $\quad$ TREE + QEDPS (ISR) 
In its original form grc4f does not support the flag for the selection of CC-03. However, a simple modification will enable it. We start with the parameters as the set for entry 4, then add an array jselg in the subroutine modmas as shown below, which corresponds to the case of $s \bar{s} c \bar{c}$. If one sets the $\mathrm{i}$-th element of jselg to "0", then one can omit this graph (see GRACE manual [8]).

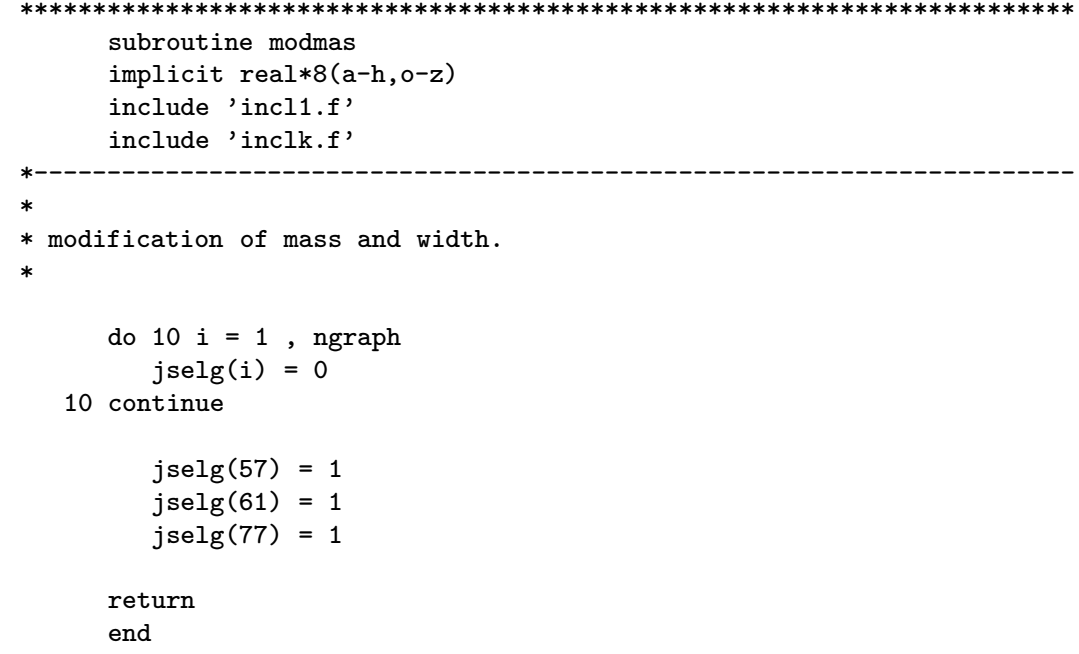

We start with the parameters as the set for entry 5 and add an array jselg in the same way as set 2 . 
Figure 1: The $\frac{d \sigma_{2}}{d M_{s \bar{s}}}$ differential distribution of the "visible" $s \bar{s}$ jets where $c \bar{c}$ jets escape detection. The centre-of-mass energy is $161 \mathrm{GeV}$. Input parameters of type 1: CC-03 no spin correlation (thin line); and type 2: CC-03 spin correlations switched on (thick line). See Appendices A, B for a complete definition of all input parameters.

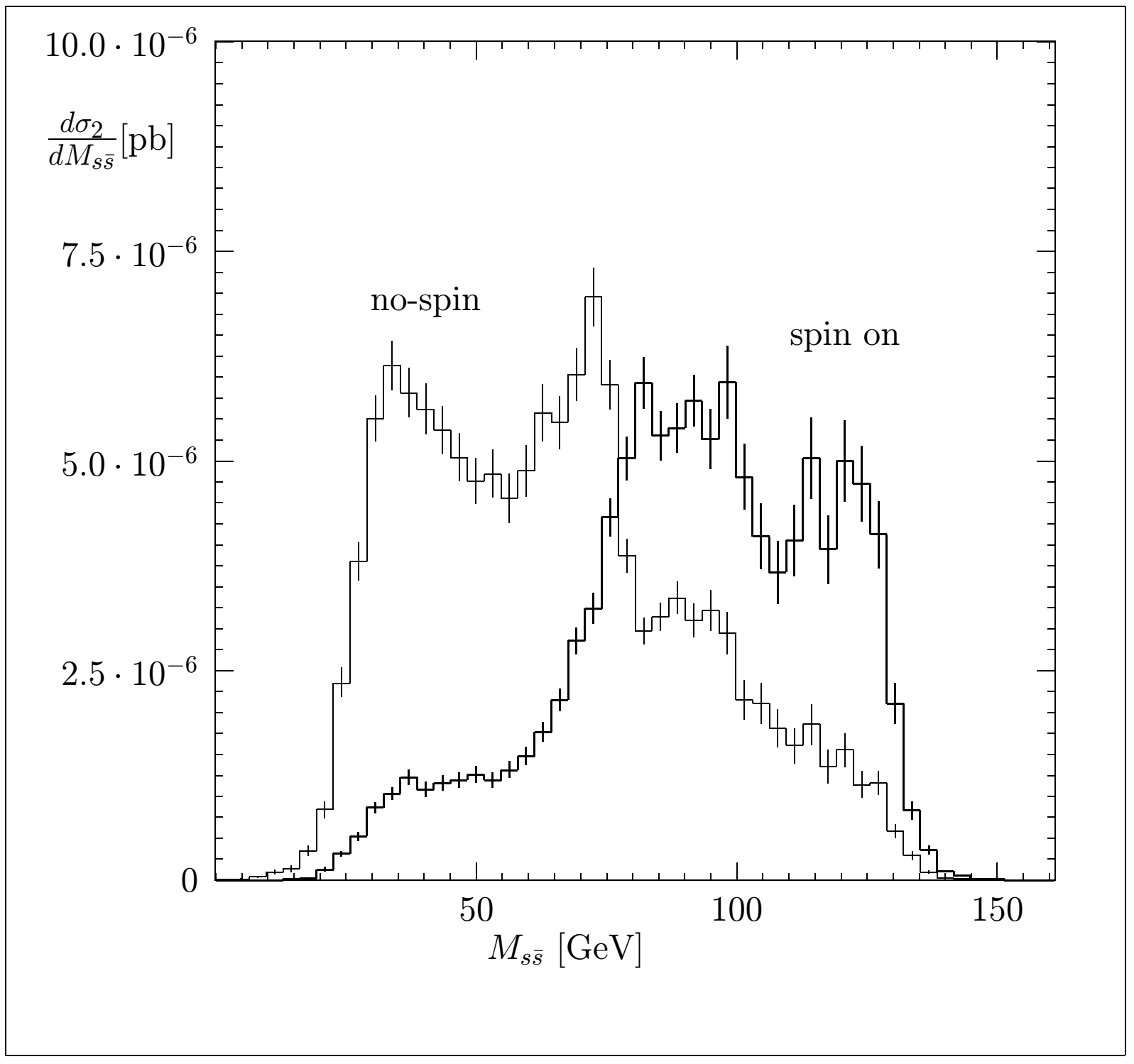


Figure 2: The $\frac{d \sigma_{2}}{d M_{s \bar{s}}}$ differential distribution of the "visible" $s \bar{s}$ jets where $c \bar{c}$ jets escape detection. The centre-of-mass energy is $161 \mathrm{GeV}$. Input parameters of type 2: CC-03 (thick line); and type 4: CC-43 (thin line). See Appendices A, B for a complete definition of all input parameters.

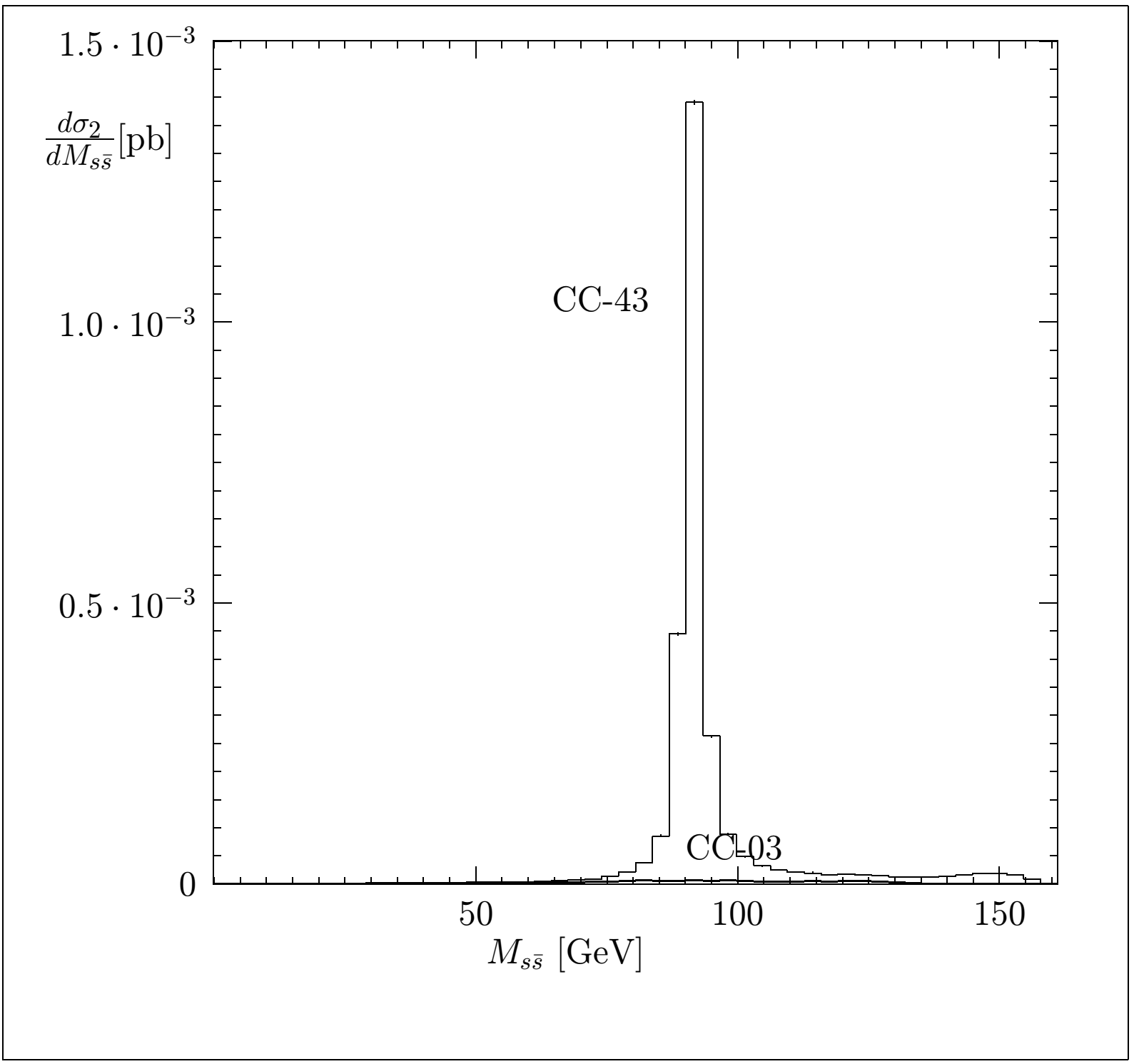


Figure 3: The $\frac{d \sigma_{2}}{d M_{s \bar{s}}}$ differential distribution of the "visible" $s \bar{s}$ jets where $c \bar{c}$ jets escape detection. The centre-of-mass energy is $195 \mathrm{GeV}$. Input parameters of type 1: CC-03 no spin correlation (thin line); and type 2: CC-03 spin correlations switched on (thick line). See Appendices A, B for a complete definition of all input parameters.

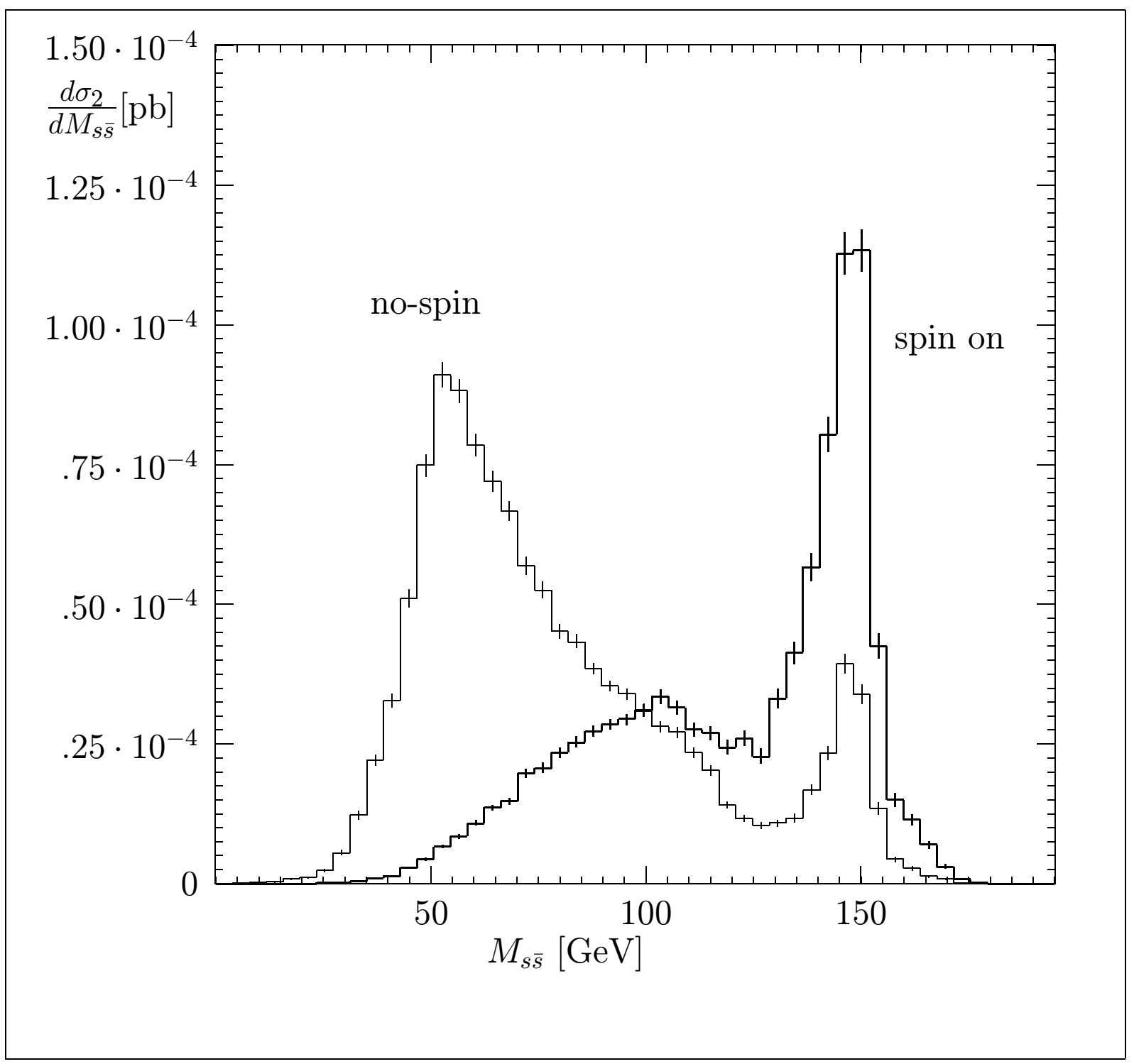


Figure 4: The $\frac{d \sigma_{2}}{d M_{s \bar{s}}}$ differential distribution of the "visible" $s \bar{s}$ jets where $c \bar{c}$ jets escape detection. The centre-of-mass energy is $195 \mathrm{GeV}$. Input parameters of type 2: CC-03 (thick line); and type 4: CC-43 (thin line). See Appendices A, B for a complete definition of all input parameters.

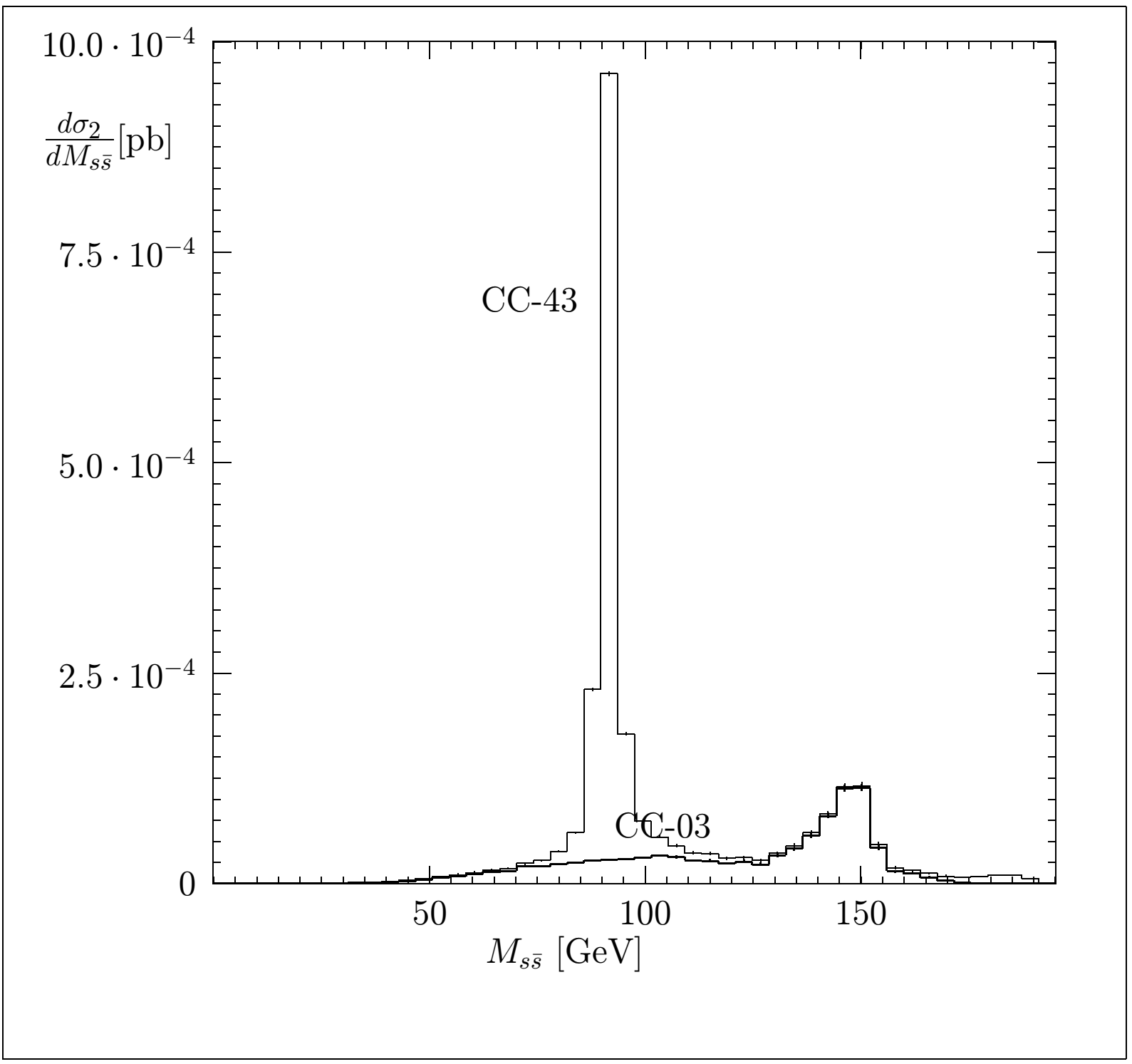


Figure 5: The $\frac{d \sigma_{2}}{d M_{s \bar{s}}}$ differential distribution of the "visible" $s \bar{s}$ jets where $c \bar{c}$ jets escape detection. The centre-of-mass energy is $350 \mathrm{GeV}$. Input parameters of type 1: CC-03 no spin correlation (thin line); and type 2: CC-03 spin correlations switched on (thick line). See Appendices A, B for a complete definition of all input parameters.

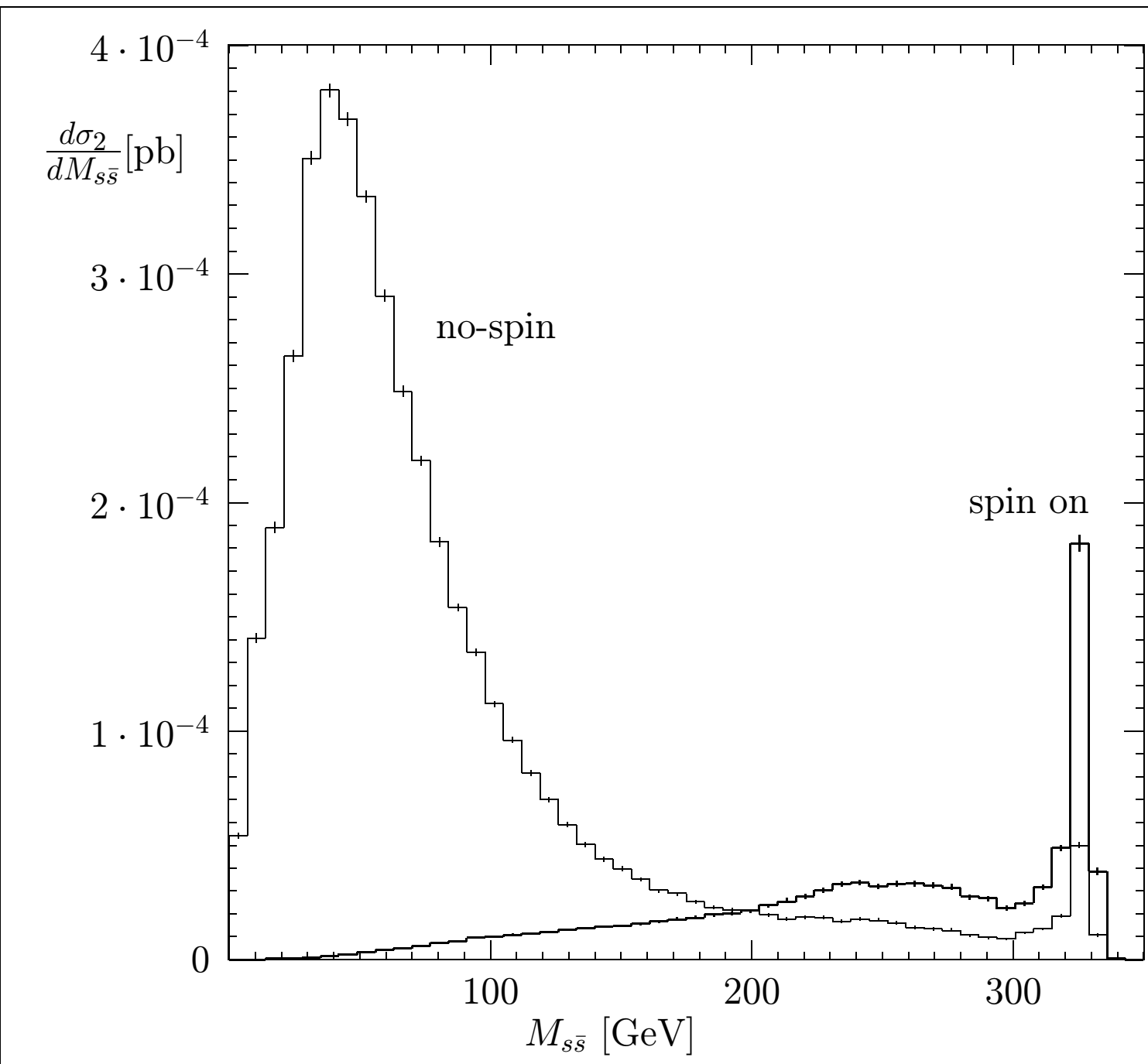


Figure 6: The $\frac{d \sigma_{2}}{d M_{s \bar{s}}}$ differential distribution of the "visible" $s \bar{s}$ jets where $c \bar{c}$ jets escape detection. The centre-of-mass energy is $350 \mathrm{GeV}$. Input parameters of type 2: CC-03 (thick line); and type 4: CC-43 (thin line). See Appendices A, B for a complete definition of all input parameters.

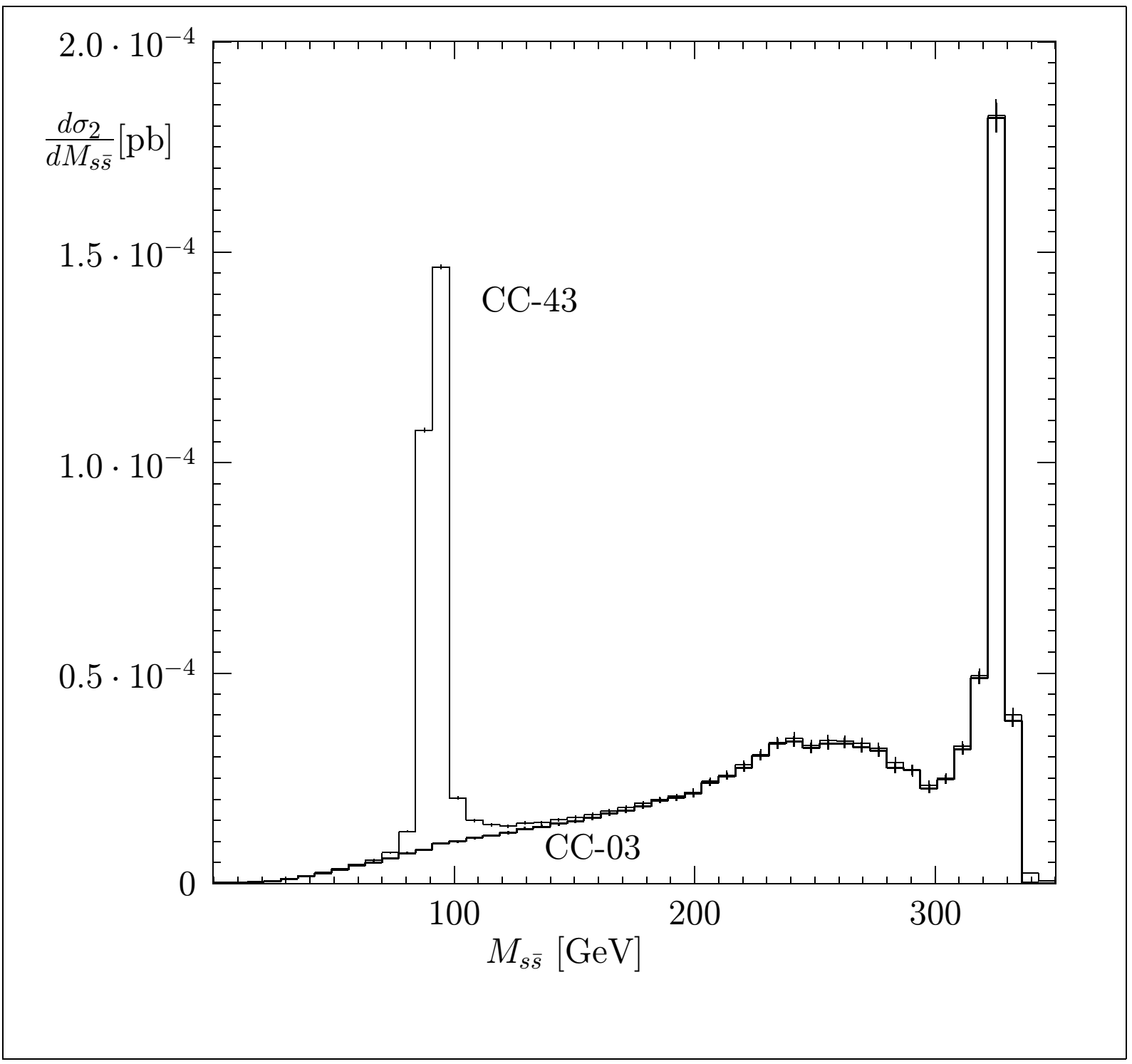

